\title{
Cutting of Graphite at Atomic and Close-to-Atomic Scale Using Flexible Enhanced Molecular Dynamics
}

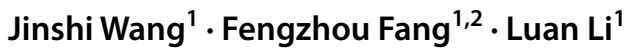

Received: 13 November 2021 / Revised: 15 January 2022 / Accepted: 20 January 2022 / Published online: 2 March 2022

(c) The Author(s) 2022

\begin{abstract}
Atomic and close-to-atomic scale manufacturing is the key technology for the production of next-generation devices with atomic precision. As an important approach of mechanical processing, cutting has evolved as a potential candidate to generate an atomically smooth surface; thus, exploring its ultimate capability is significant. In this paper, single-crystal graphite, whose lattice structure and chemical bond property are of representation for demonstration, is selected to study the mechanism of atomic layer removal using molecular dynamics. A localized workpiece, which is dynamically updated on the basis of the tool position, is used to improve the computation efficiency. The principle and bullet points of this modeling method are first introduced, followed by a series of simulations under various undeformed chip thicknesses and tool edge radii. In addition, different potentials for the tool-workpiece interaction are tested, and the effect on the material response is presented. Based on the analysis of deformation, the number of carbon layers removed, and cutting forces, the chip formation mechanism and further understanding of the controllability of cutting at atomic and close-to-atomic scale can be achieved.
\end{abstract}

Keywords Atomic and close-to-atomic scale $\cdot$ Cutting $\cdot$ Enhanced molecular dynamics $\cdot$ Graphite $\cdot$ ACSM

\section{Introduction}

More and more critical devices and structures in microelectronics and optical engineering require a feature size or surface finish at the sub-nanometric level. For quantum technology, a nearly perfect material with atomic-level integrity is crucial. These devices and structures show great application potential in the advancement of manufacturing technology, which is the key to upgrading products and improving daily life. Manufacturing has been evolving from the handicraft-based phase to the machine-based phase, with a dramatic increase in the precision from millimeter and submillimeter to micrometer and nanometer level, and it is developing toward the next generation, namely, atomic

Fengzhou Fang

fzfang@tju.edu.cn

1 State Key Laboratory of Precision Measuring Technology and Instruments, Laboratory of Micro/Nano Manufacturing Technology (MNMT), Tianjin University, Tianjin 300072, China

2 Centre of Micro/Nano Manufacturing Technology (MNMT-Dublin), University College Dublin, Dublin 4, Ireland and close-to-atomic scale manufacturing (ACSM) [1-3]. In ACSM, materials are added, removed, or transported at the atomic scale in a controllable manner, which might be achieved through mechanical, optical, chemical, or any other approaches. As a typical mechanical process, cutting is an important method in precision engineering, whose fundamental theory and application have been investigated for a long time. In the past decades, nanometric cutting has undergone fast development, and it became a critical approach for ultra-precision machining [4]. The characteristic material removal mechanism in nanometric cutting is dominated by extrusion, which is significantly different from shearing in conventional cutting $[5,6]$. To be a candidate of ACSM, chip formation and surface generation have to be clarified when the undeformed chip thickness (UCT) is further reduced to the atomic scale. In particular, the condition of single atomic layer removal and its dependence on the process parameters have great significance on the cutting theory and practice.

At present, some studies on the above-mentioned issues have been conducted using molecular dynamics (MD) to simulate atomic-scale cutting on monocrystalline copper. A critical phenomenon, namely, the atomic size effect, shows that chip formation is dependent on the ratio of cutting depth to atomic radius [7]. This ratio influences the 
relative position between the atoms on the top layer of the workpiece and at the bottom of the tool and the force diagram. Consequently, distinct material removal regimes successively occur with the increase in the UCT, including no material removal, no continuous material removal, and continuous material removal. The limit of single atomic layer removal can be achieved theoretically when the UCT is approximately $2 \AA$, reaching the highest repeatability on the copper (111) surface. A novel chip formation mechanism under atomic and close-to-atomic scale (ACS), namely, shear stress-driven dislocation motion, is proposed, which is different from extrusion and shearing in nanometric and macro cutting, respectively. In addition, only elastic deformation occurs during ACS cutting. The absence of plastic deformation is critical for a high-quality machined surface with atomic integrity. Based on analysis of stress field and cutting force, the effect of edge radius and chip formation mechanism on the number of atomic layers removed is understood [8]. A model predicting the minimum chip thickness is proposed based on which the critical edge radius for single atomic layer removal is determined to be $2.7-4.2 \mathrm{~nm}$ [9]. In these studies, the workpiece $(\mathrm{Cu})$ atoms interact with one another through the same metallic bond, whereas cutting would be different if more than one kind of atomic bond exist, which is explored in this paper.

Graphite is a material that has covalent bonds in the carbon layers and van der Waals bonds between them. This crystal structure is suitable for investigating the atomic layer removal process. Meanwhile, graphite becomes a critical material used in precision electrode, sealing components of aeroengine, fuel cell plate, and neutron slower because of its outstanding electrical conductivity and chemical and thermo stability. The quality of the graphite surface strongly influences product functionality. For example, surface roughness at the micrometer level is required for cold electron emitters [10]. Reducing the roughness can prolong the life of solid rocket motor nozzles [11] and improve the chemical vapor deposition of $\mathrm{SiC}$ on nuclear fuel cladding [12]. In addition, a nanometric graphite surface is necessary to obtain highly efficient secondary electron emission in the research of plasma [13]. An ultrasmooth graphite surface with atomic-level integrity can also be expected to be necessary for various applications; thus, manufacturing becomes a critical issue.

Mechanical processing is a major approach for graphite production, which has been developed over 30 years. As a brittle material, surface fracture and tool wear remain a challenge in machining. Systematical studies have been conducted on chip formation, cutting forces, and the effect of process parameters under the UCT of the submillimeter level $[14,15]$. However, macro cutting can neither achieve a smooth graphite surface nor reflect the cutting mechanism in nanometric or ACS. A few reports about the ultra-precision diamond turning experiment have been found, in which a surface roughness of $40 \mathrm{~nm}$ in $R a$ could be achieved. Therefore, micro cracks still occur on the machined surface, which is due to serious abrasive wear on the flank face $[16,17]$. Although boron doping and nitrogen environment are used to prolong the tool life [18, 19], obtaining a crack-free surface is still difficult. In brief, either in the ACS or nanoscale, fundamental studies to understand graphite deformation during cutting are lacking. In particular, numerical simulation from the atomic perspective is absent. Therefore, the MD is used in this work to investigate the cutting mechanism. A novel method using a dynamic workpiece is upgraded on the basis of previous versions to improve simulation efficiency [20, 21]. Chip formation and the effects of UCT, edge radius, and potential function are analyzed for ACS cutting. A large model is built to demonstrate the capability of the novel simulation method, accompanied by a brief discussion on nanometric cutting. The results show that single-crystal graphite exhibits outstanding machinability in the formation of atomic-level machined surfaces.

\section{Method}

\subsection{Flexible Enhanced Molecular Dynamics (f-EMD)}

Efficient simulation technology is always expected in the MD study of cutting either at the ACS or nanometric scale. Using a localized workpiece that is updated dynamically to follow the tool motion can significantly reduce the atom number and speed up the simulation. This is achieved by forward extension and backward truncation of the workpiece at specific instances based on the tool motion trajectory before cutting. Furthermore, the initial coordinates of atoms in the extended zone should be carefully assigned to make the lattice structure and thermodynamic status continuous across the boundary between the existing workpiece and newstitched material. Otherwise, a stress gradient would occur, and an artificial pressure wave would be triggered. However, the length of the extended zone was always the same as that of the initial workpiece in previous studies (Fig. 1a). It reduces not only the flexibility but also the superiority of EMD because a shorter extension length could further improve the simulation efficiency.

In this paper, the traditional EMD is developed to resolve the above-mentioned problem. As shown in Fig. 1b, the first step is to build and relax a precursor model with periodic boundary condition (PBC) in the length $(x)$ and width $(y)$ dimensions. Notably, the two sides of the model are correlated with each other rather than independent if the PBC is applied in the corresponding dimension. Then, the precursor is duplicated to generate the initial workpiece. Considering that all of the red boundaries in Fig. 1b are correlated with 
Fig. 1 Schematic illustration of flexible enhanced molecular dynamics. a In the traditional EMD, the extended zone and initial workpiece have the same length. b A variable extension length is adopted in this work for higher flexibility and efficiency

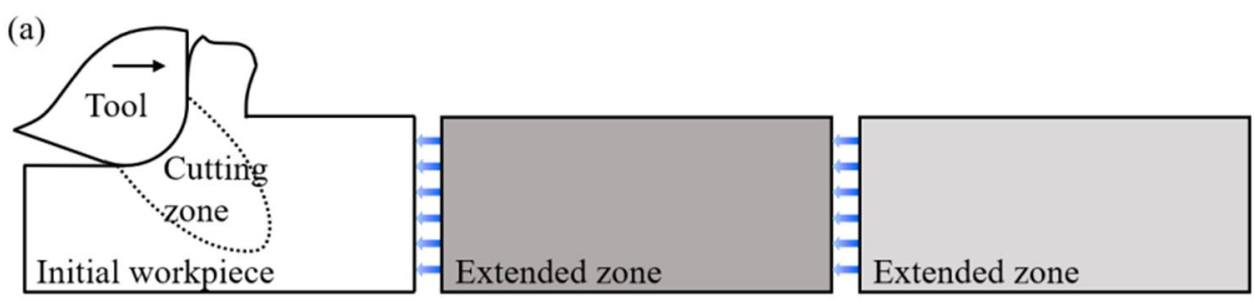

(b)
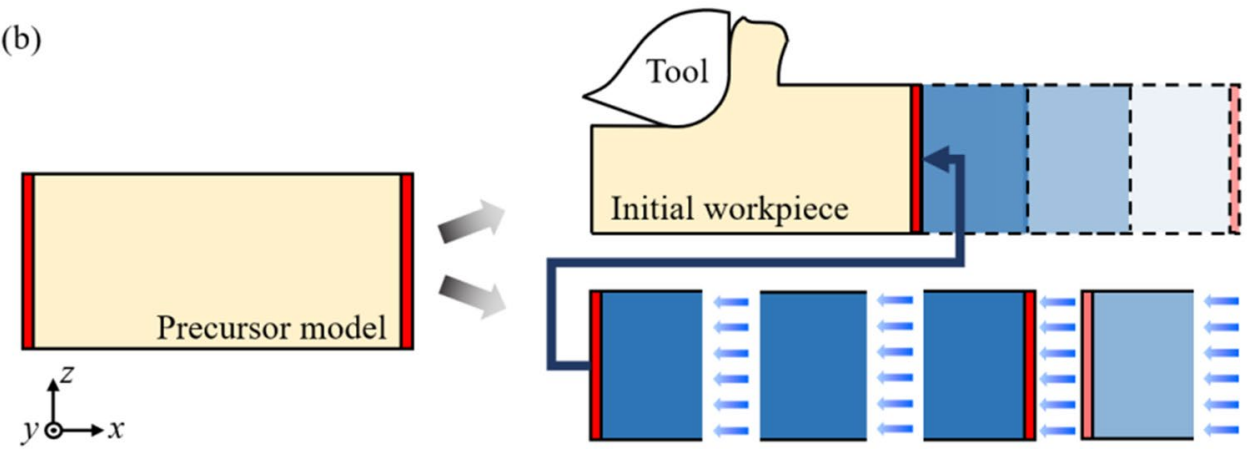

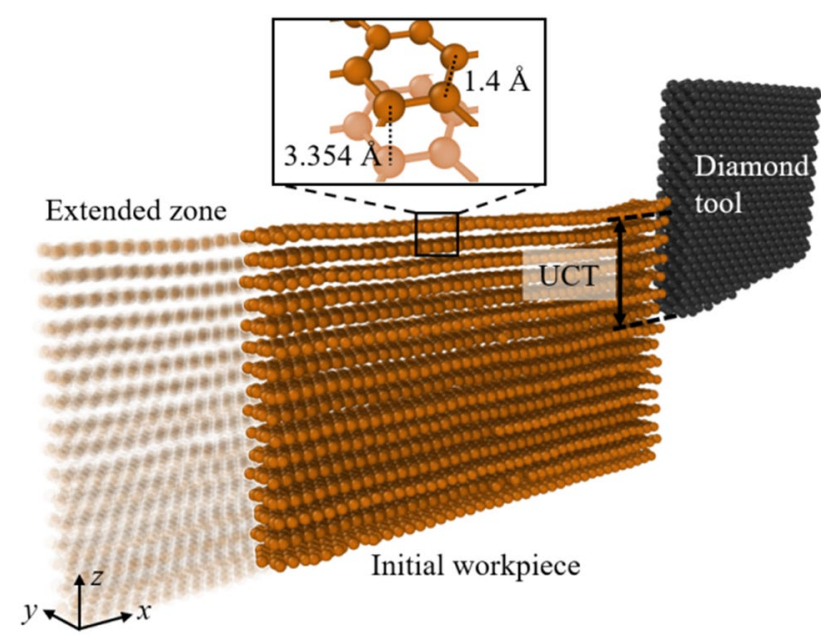

Fig. 2 Simulation model of ACS cutting on single-crystal graphite

PBC, a smooth connection between the extended zone and the existing workpiece is ensured. Finally, the model used for the extension is sliced into equal pieces to obtain a variable length. These pieces would be stitched to the workpiece one by one in order. Such pieces are obtained from the same material; thus, their boundaries are automatically correlated, which is critical to achieving the adjustment of extension length. The f-EMD is realized based on the Large-scale Atomic/Molecular Massively Parallel Simulator (LAMMPS) [22], and a user interface is developed by Qt.

\subsection{Simulation Setup}

Figure 2 shows the f-EMD model of ACS cutting on graphite. The length, height, and thickness of the initial workpiece are 10,6 , and $0.8 \mathrm{~nm}$, respectively. $\mathrm{PBC}$ is applied in the $y$-dimension, along which the model scale is two times the lattice constant. Graphite unit cell contains four atoms, in which the bond length of the six-member ring is $1.4 \AA$, and the interlayer distance is $3.354 \AA$. The interaction among the workpiece atoms is described by the AIREBO potential function [23] for $\mathrm{C} / \mathrm{H}$ systems, which has been used in the MD study of graphite [24]. This potential consists of three terms: the reactive empirical bond order (REBO) term for short-range interaction, the Lennard-Jones (LJ) term for long-range intermolecular interaction, and the torsional term for the dihedral angle, which is a four-body interaction. However, the potential that is suitable for graphite-diamond interaction has not been reported. Therefore, we first compare the results using two kinds of tool-workpiece interactions, namely, LJ and analytical bond order potentials (ABOP [25]), to select the suitable potential. Then, systematic simulations are conducted to reveal the chip formation under the UCT of $0.5,0.7,1.25$, and $2.5 \mathrm{~nm}$, which corresponds to $1,2,4$, and 7 atomic layers, respectively. Various edge radii $(0.1$, 2 , and $5 \mathrm{~nm}$ ) are considered to study the size effect. The diamond tool is treated as a rigid body with a nominal rake angle of $0^{\circ}$ and a clearance angle of $12^{\circ}$. The cutting speed 
is $50 \mathrm{~m} / \mathrm{s}$, and the timestep is $1 \mathrm{fs}$. The workpiece is stabilized at room temperature using the canonical ensemble.

\section{Results and Discussion}

\subsection{Influence of Tool-Workpiece Interaction Potential}

As shown in Fig. 3, material deformation during ACS cutting of graphite is influenced by the tool-workpiece interaction potential. The ABOP function exhibits strong attraction, wherein the carbon layers are firmly stuck to the cutting edge, thereby resulting in a hump-like deformation of the atomic layers being removed. The hump moves along the cutting direction in front of the tool, and the lattice in this zone maintains the ring structure. However, using the ABOP may not be appropriate in this study. It is known that the cutting forces in tangential and thrust directions should have different magnitudes, which depend on the UCT and edge radius. By contrast, the two forces have the same value, which fluctuates violently near zero. The fluctuation is even larger than the force in the ACS cutting of copper [9], but at least the tangential force

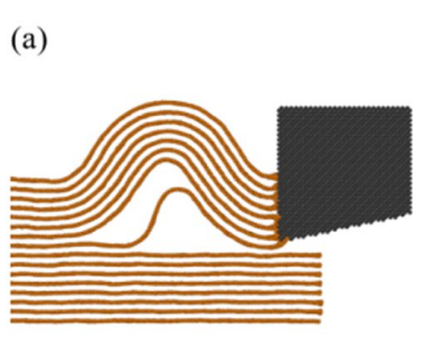

ABOP

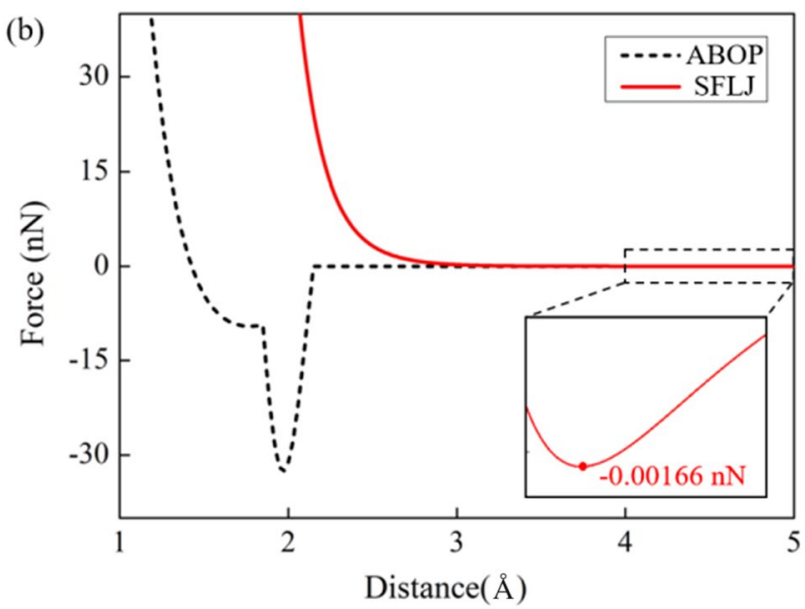

Fig. 3 Effect of potential function. a Simulation snapshots where the edge radius is $0.1 \mathrm{~nm}$ and the UCT is $2.5 \mathrm{~nm}$. b Two-body force curves of different potentials and the negative value stands for attraction. (The SFLJ parameters are referred to those in Table 1) should be smaller because graphite provides better lubrication than copper. A sudden adhesion and collapse can be observed once the hump gets close to the rake face, and a large number of workpiece atoms diffuse to the interstitial sites of the diamond lattice. (Relative positions among the diamond atoms never change). These phenomena indicate that artificial results arise when using an inappropriate potential function.

Material deformation and chip formation show a dramatic change after applying the $\mathrm{LJ}$ potential. The removed atomic layers are bent upwards and then slide along the rake face. The strong adhesion and violent force fluctuation disappear, and the effect of edge geometry on the force components occurs as discussed below. It is confirmed in Fig. $3 b$ that the ABOP has a stronger attraction at a short distance, which makes the workpiece atoms difficult to escape once they are close to the tool. By contrast, attraction is weak, and it acts at a longer distance in the LJ potential used in this study. By checking the atom coordinate, the minimum chip-tool distance falls into the range of repulsive force. Therefore, the force status of the chip atoms directly interacting with the tool atoms is different for the two potentials, which finally leads to different deformations. On the other hand, the LJ potential should be carefully tuned to avoid another artificial effect; that is, many carbon layers in the machined zone are attracted and raised collectively by the flank face. This effect is due to the abrupt truncation of the potential at the cutoff radius, which results in discontinuous energy and enhanced attractive force. Therefore, the shifted force LJ (SFLJ) potential is used, whose energy is always continuous. The distance and energy units of the SFLJ function are set to be the same as those in the LJ term of AIREBO potential, and the cutoff radius is 1.5 times the distance unit according to the literature [26]. Notably, an energy unit $(2.84 \mathrm{eV})$, which is three orders higher than that used in this work, was used in the study of carbon nanotube [27]. The deep potential well is close to the $\mathrm{C}-\mathrm{C}$ single covalent bond energy $(\sim 3.6 \mathrm{eV})$, but it is quite different from the interaction between graphite and diamond surfaces from two objects; thus, the energy unit has to be reduced to eliminate the artificial effect. A similar amplification also occurs in the maximum attraction force, which increases from $-0.00166 \mathrm{nN}$ to approximately $-1 \mathrm{nN}$. This phenomenon provides instructive perception to optimize the potential function. In principle, many potentials are not designed for formulating the interaction between surfaces but are used in the nanometric cutting simulation. Although this issue has not been explicitly concerned and seems to be not critical in those cases, it should

Table 1 Potential function of tool-workpiece interaction

\begin{tabular}{llll}
\hline Type & Energy unit $(\mathrm{eV})$ & Distance unit $(\AA)$ & $\begin{array}{l}\text { Cutoff radius } \\
(\AA)\end{array}$ \\
\hline SFLJ & $2.84 \times 10^{-3}$ & 3.4 & 5.1 \\
\hline
\end{tabular}


not be neglected at ACS. Details about the tool-workpiece potential are listed in Table 1.

\subsection{Enhancement in the Simulation Efficiency by f-EMD}

The accumulated workpiece length and cutting distance are kept constant to study the influence of extension length on simulation time. In each test, an initial workpiece with $10 \mathrm{~nm}$ length is first built, and it could be extended as the tool advances. As shown in Fig. 4, the larger the model update number, the shorter the piece of material used for workpiece extension. The tests are run on a workstation with the Intel Xeon processor and $30 \mathrm{CPU}$ cores. It is clear that a smaller extension length actually reduces the average atom number during the whole simulation and higher efficiency can be achieved. In this work, a $21 \%$ reduction in the total time is reached, and the simulation time almost keeps a linear decrease with the atom number. We also compare the computing time using three methods: conventional modeling with a global workpiece, traditional EMD (Test-4 in the figure), and the f-EMD developed in this study. The time consumption of conventional modeling is $103 \mathrm{~min}$, which is reduced to $98 \mathrm{~min}$ when using the traditional EMD. However, this difference is not significant because the moderate atom number in this study is not large enough to forcefully demonstrate the power of the traditional EMD. In addition, the variable extension length of f-EMD can further improve the simulation efficiency, and its merit would be more remarkable when the model scale increases. In tests using the traditional EMD, the workpiece height was enhanced to $200 \mathrm{~nm}$ with a UCT of $50 \mathrm{~nm}$ and edge radius of

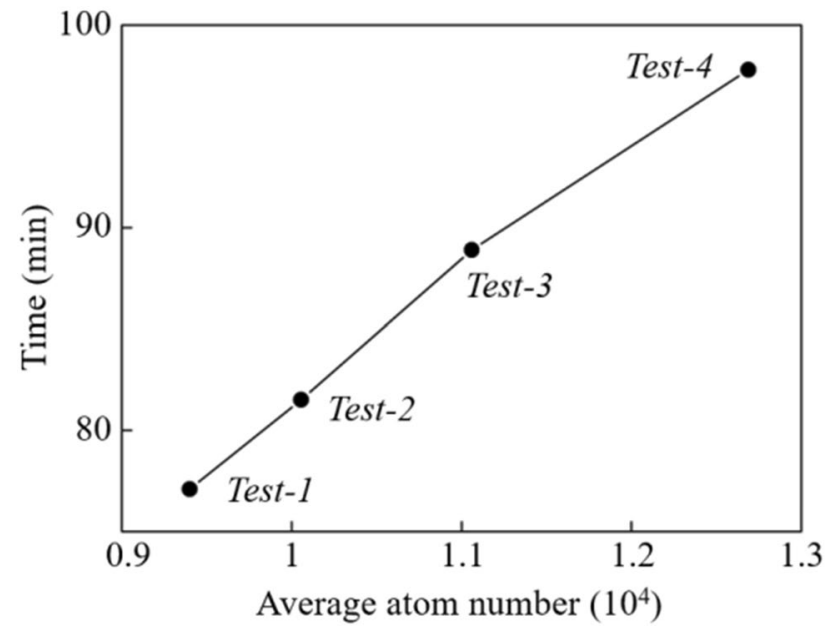

Fig. 4 Variation of simulation time with average atom number. From test- 1 to test-4, the model update number is $4,3,2$, and 1 with an extension length of 2.5,3.3, 5, and $10 \mathrm{~nm}$, respectively. (Edge radius: $0.1 \mathrm{~nm}$, UCT: $0.7 \mathrm{~nm}$ ) approximately $70 \mathrm{~nm}$. It can be expected that the simulation size can be further improved using the f-EMD.

\subsection{Mechanism of ACS Cutting of Graphite}

The distinctive atomic bond property makes the chip formation of graphite quite different from other typical materials, such as silicon and copper. In nanometric cutting, a highly localized zone is found in the vicinity of the tool edge where the workpiece lattice becomes totally amorphous and extrusion occurs. As shown in Fig. 5, this amorphization disappears because the weak van der Waals bonds are easy to break, which is the dominant manner to dissipate the energy introduced by the tool. Different from ACS cutting of copper, no dislocation behavior is observed, and the chip is directly stripped from the substrate at a wedge-like region in front of the tool. Despite the large deformation, the covalent bonds in each atomic layer are not broken; thus, the lattice topology in the chip does not change. Similarly, the machined surface shows an ultra-high quality without any subsurface deformation and lattice defect. As can be seen later, this finding is irrespective of the UCT and edge radius, which has great significance in achieving surface integrity at the atomic level.

Figure 6 shows the snapshots from various cutting conditions. When an absolutely sharp tool ( $0.1 \mathrm{~nm}$ edge radius) is used, the chip is formed via simple deformations, such as bending and sliding (Sect. 3.1). Carbon layers in the range of UCT can be totally removed, and material deformation under the tool edge is slight. However, the atomic layers removed may exceed the theoretical number, as in the case of $1.25 \mathrm{~nm}$ UCT. This result is due to the thermal vibration of workpiece atoms, particularly those at the bottom of the

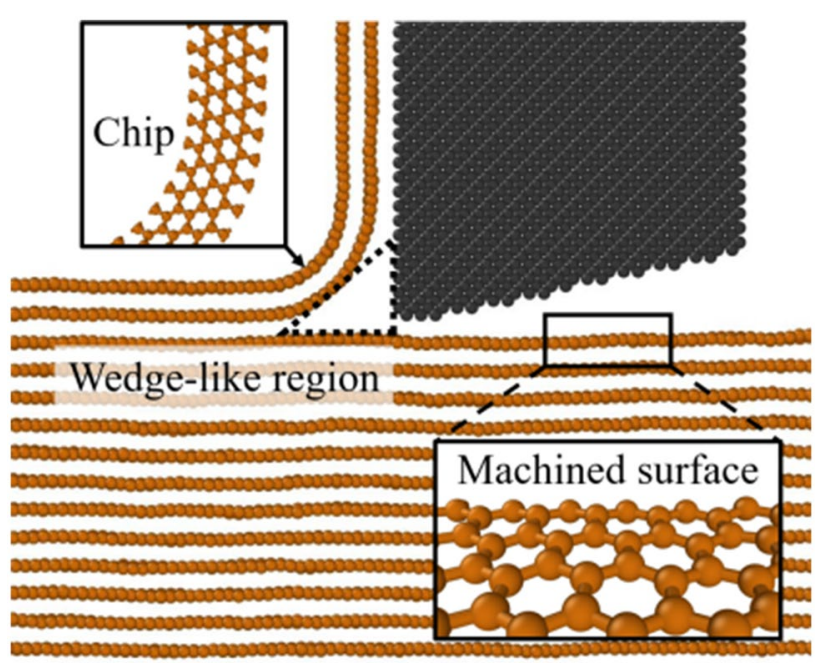

Fig. 5 Chip formation and surface generation during ACS cutting of graphite. (Edge radius: $0.1 \mathrm{~nm}, \mathrm{UCT}: 0.7 \mathrm{~nm}$ ) 


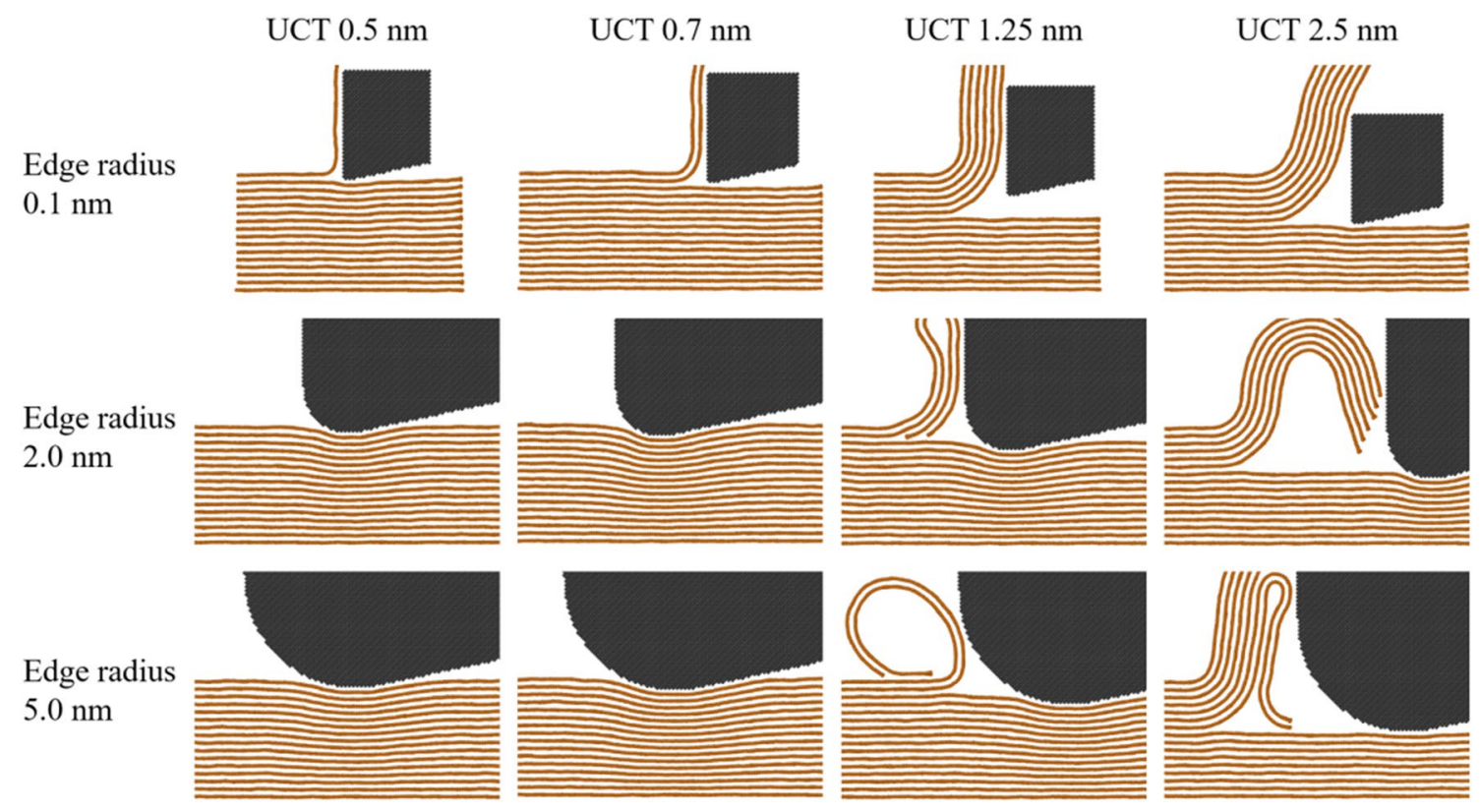

Fig. 6 Cutting with various edge radii and UCTs

cutting edge. If these atoms occasionally vibrate upwards to the free surface at the moment when the tool touches the workpiece, then extra atomic layers would be removed. On the contrary, if they vibrate downwards, then some layers might be left. This thermal effect introduces uncertainty of the atomic layer removal number (ALRN), which can be alleviated by reducing the temperature. As the edge radius increases, the effective rake angle becomes negative, and chip deformation becomes complex. The carbon layers are folded or severely curved. In addition, not all the layers in the range of UCT would be removed, and uncut atomic layers would be elastically depressed, similar to the material branch flow in nanometric cutting. However, atoms in the chip and beneath the edge still maintain the ring structure without any phase transformation or intersection of the carbon layers. This should be a common feature of ACS cutting on materials with a weak interlayer bond.

As shown in Fig. 7a, single atomic layer removal is possible theoretically, but it can only be achieved under extreme conditions, where the tool has atomic-level sharpness. The minimum ALRN is larger than one under the UCT of $1.25 \mathrm{~nm}$ when the edge radius increases to 2 and $5 \mathrm{~nm}$. Therefore, making a sharp tool to achieve the limit of ACS cutting is challenging. In addition, the discrepancy in the ALRN is large under small UCT, and it decreases under large UCT despite different chip morphologies. This insensitivity to the edge radius is due to the increase of effective rake angle with UCT, which improves the controllability of ACS cutting practice. Figures $7 \mathrm{~b}-\mathrm{d}$ present cutting force analysis, where the raw data are first smoothed to erase the high-frequency signal. The force curves rise at the initial stage, then fluctuate near a stable value. It is interesting that the principal force is nearly zero and is weakly influenced by the process parameters, which is a unique phenomenon resulting from the outstanding lubrication of graphite. In general, the thrust force increases with the edge radius. For sharp tools, it is not sensitive to the UCT. For large edge radii, it initially increases with the UCT because more atomic layers are depressed when no chip formation occurs. When the ALRN is nonzero, a portion of strain energy is released by the chip; thus, the subsurface deformation and thrust force decrease. The thrust force would be significantly reduced if the ALRN is equal to the theoretical number, where the elastic deformation beneath the edge is slight. This finding indicates the force drop shown in Fig. $7 \mathrm{~d}$ under the condition of 5-nm edge radius and 2.5-nm UCT.

Finally, nanometric cutting simulation is conducted using a large model, in which the UCT and edge radius are 20 and $40 \mathrm{~nm}$, respectively. As shown in Fig. 8, the chip formation mechanism is similar to that at ACS. For example, the chip is formed via complex folding deformation without lattice defects in the carbon layers. Atomic-level surface integrity can be achieved without subsurface deformation. In addition, the thrust force is evidently larger than the principal force, which indicates that the flank wear is the primary issue observed in the experiment [28]. However, such a large increase in the UCT does not lead to a considerable increase in the thrust force compared with ACS cutting. More details can be revealed by the stress field shown in Fig. 8c. Although the hydrostatic stress concentrates under the tool edge, which is ordinary in nanometric cutting, neither high-pressure 
(a)

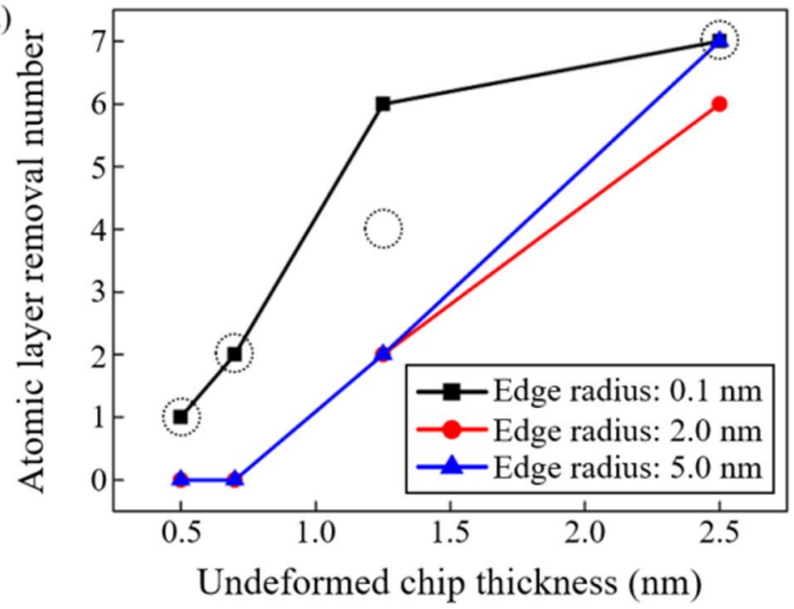

(c)

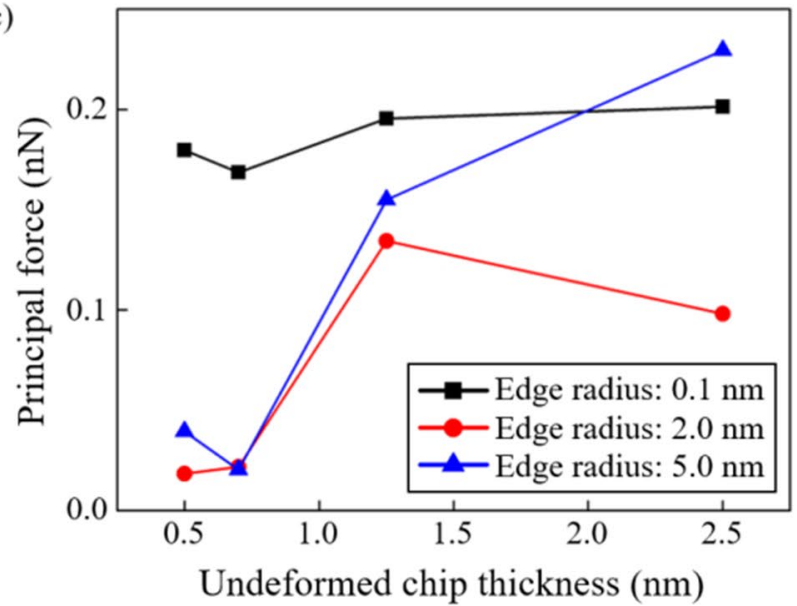

(b)

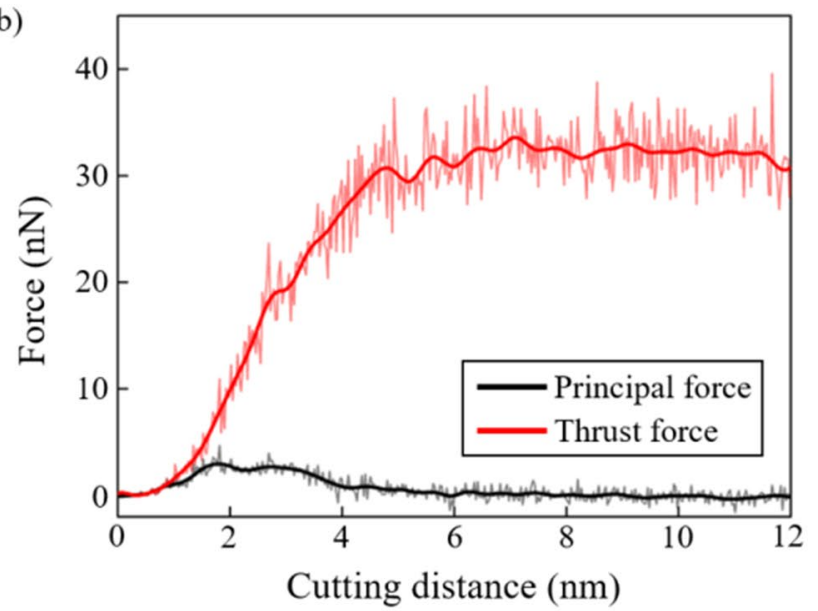

(d)

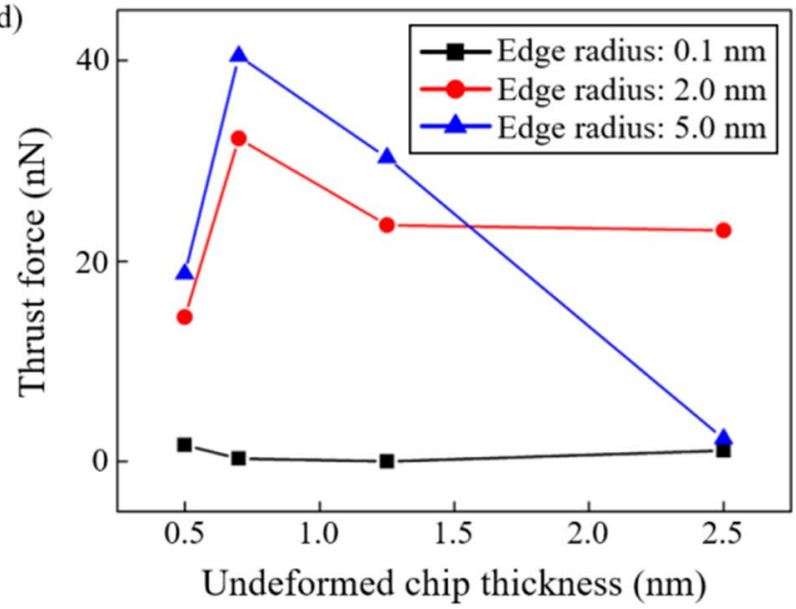

Fig. 7 Atomic layer removal number and cutting forces. a The theoretical numbers are marked by dashed circles. b-d The cutting distance is long enough to evaluate the forces in the stable stage

phase transition nor shear band is found. Therefore, even in nanoscale, graphite exhibits a distinctive manner of chip formation by folding. The first principal and maximum shear stresses show a random pattern, so other three stress components in the $x-z$ plane are further considered. It is obvious that the tensile stress (positive value) along the thrust and cutting directions is primarily distributed in the chip and subsurface, respectively. Its strength is several $\mathrm{GPa}$, but no fracture is found in the workpiece, because the stress direction is always parallel to the carbon layers (see the enlarged view of the circled region), and the tensile strength of graphene can be larger than $100 \mathrm{GPa}$ in this direction [29]. By contrast, the tensile stress normal to the carbon layers is lower than the critical value of approximately $700 \mathrm{MPa}$ [24]. This stress state prevents crack formation and propagation. Furthermore, the pattern of shear stress $S x z$ seems to be complementary to that of the normal stress $S z z$ in the chip, with lower strength. However, the simulation has not shown the brittle failure observed in experiments, which may be a result of material defects (vacancy, grain boundary, impurity, etc.) or a tool velocity non-parallel to the carbon layers.

\section{Conclusions}

Atomic and close-to-atomic scale cutting of graphite, a typical representative of layered structure material, is studied in this paper. An advanced simulation method is developed, based on which the cutting mechanism is revealed. The results show that single-crystal graphite is a potential candidate for controllable material removal of several or even single atomic layers with ultra-high machined surface integrity. The main conclusions are summarized as follows:

(1) Flexible enhanced molecular dynamics is further developed using a variable extension length to achieve high simulation efficiency. A remarkable reduction in 
(a)

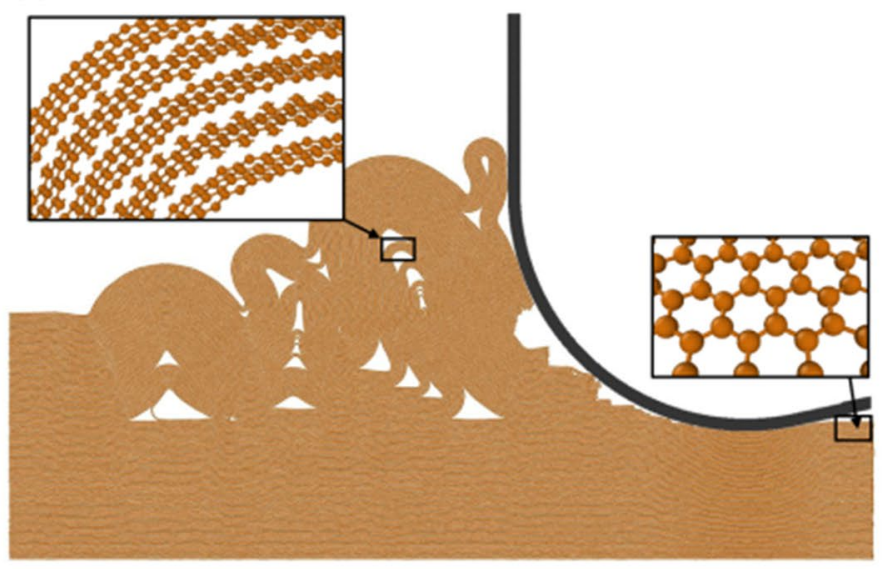

(b)

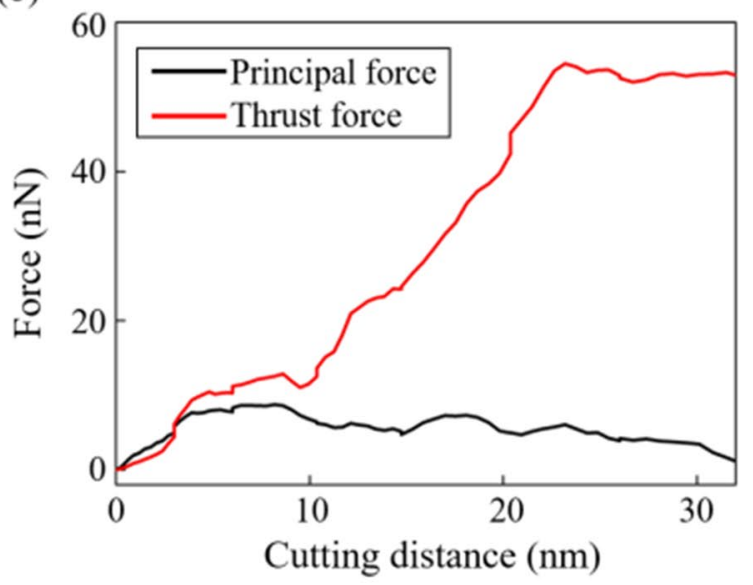

(c)

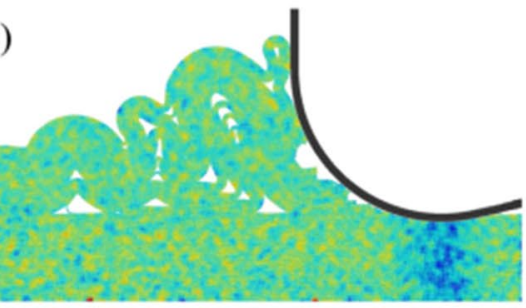

$-5=5$

Hydrostatic stress (GPa)

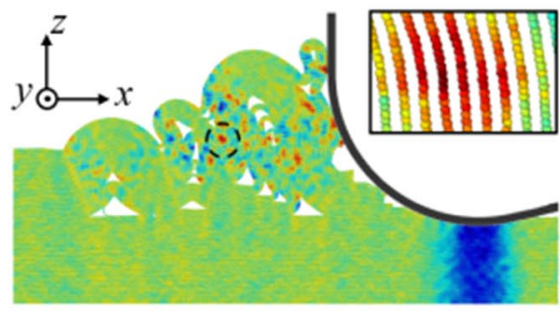

$-5$

$\mathrm{Szz}(\mathrm{GPa})$

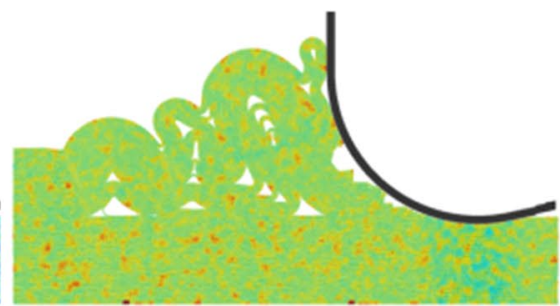

$-10=10$

First principal stress (GPa)
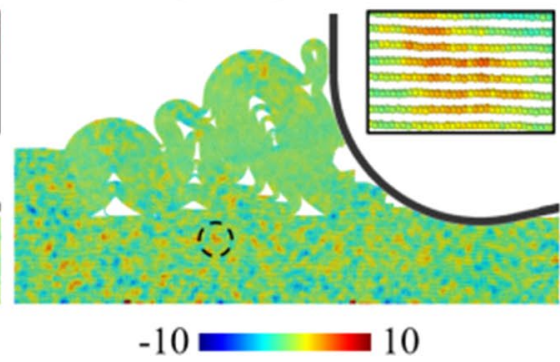

$\operatorname{Sxx}(\mathrm{GPa})$

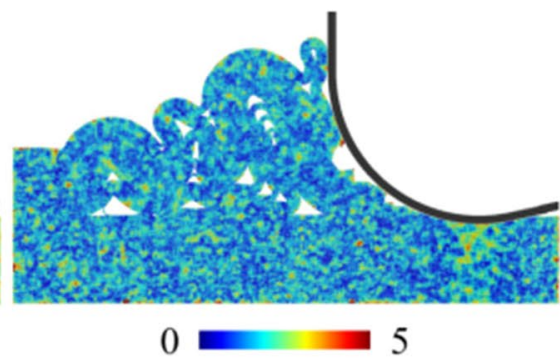

Maximum shear stress (GPa)
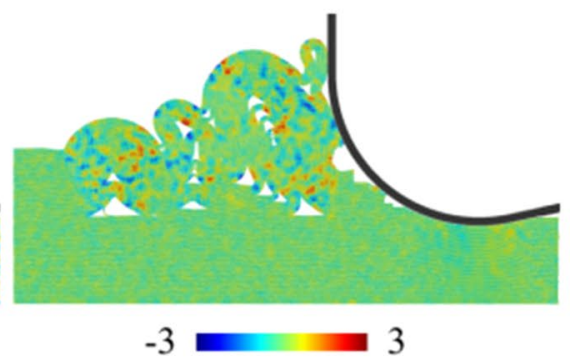

$\mathrm{Sxz}(\mathrm{GPa})$
Fig. 8 Nanometric cutting of graphite. The cutting speed is enhanced to $100 \mathrm{~m} / \mathrm{s}$ to accelerate the simulation. a Chip deformation. (The lower right enlarged view is the top carbon layer of the machined

computing time is not achieved by the traditional EMD because of the moderate atom number, but it can be successfully achieved by reducing the extension length. This evolution would be beneficial as the model scale increases.

(2) The potential function for the tool-workpiece interaction has a strong influence on ACS cutting. First, the shifted force LJ potential is more suitable than the ABOP. Second, an excessively large energy unit should not be used. These settings aim to avoid the strong attraction force, which depends on the assumption that the force between diamond and graphite surfaces is weaker than the covalent bond in the carbon layer. surface). b Cutting forces. c Stress field calculated using a statistical cube with 2-nm edges

(3) The diversity of the chemical bond in graphite results in a unique cutting process. For a very sharp tool, the chip is formed by bending and sliding along the rake face, and as the edge becomes blunt, folding deformation is dominant. In the chip and machined surfaces, atoms maintain the ring structure in carbon layers without a lattice topological defect, which is important for atomic precision manufacturing.

(4) The extreme sharpness of the tool is essential to the single atomic layer removal and is a great challenge in practice. A thermal-induced uncertainty in the number of atomic layers removed is observed, which indicates the necessity of temperature control. The princi- 
pal force is always low and insensitive to the process parameters, and the thrust force is influenced by the edge radius, undeformed chip thickness, and thermalinduced uncertainty. Experimental studies are required in the future to validate the theoretical results.

In addition, fundamental issues of nanometric cutting of graphite, such as brittle fracture, tool wear, and effect of process parameters (such as rake angle and cutting speed), are still open and valuable to investigate using the f-EMD.

Acknowledgements This work was supported by the Science Challenge Project (No. TZ2018006-0201-01) and National Natural Science Foundation of China (Nos. 52035009, 61635008).

\section{Declarations}

Conflict of interest The authors declare that they have no conflicts of interest.

Open Access This article is licensed under a Creative Commons Attribution 4.0 International License, which permits use, sharing, adaptation, distribution and reproduction in any medium or format, as long as you give appropriate credit to the original author(s) and the source, provide a link to the Creative Commons licence, and indicate if changes were made. The images or other third party material in this article are included in the article's Creative Commons licence, unless indicated otherwise in a credit line to the material. If material is not included in the article's Creative Commons licence and your intended use is not permitted by statutory regulation or exceeds the permitted use, you will need to obtain permission directly from the copyright holder. To view a copy of this licence, visit http://creativecommons.org/licenses/by/4.0/.

\section{References}

1. Fang FZ, Zhang N, Guo DM et al (2019) Towards atomic and close-to-atomic scale manufacturing. Int J Extreme Manuf 1:012001

2. Fang FZ (2020) On atomic and close-to-atomic scale manufacturing development trend of manufacturing technology. China Mech Eng 31(9):1009-1021

3. Fang FZ (2020) Atomic and close-to-atomic scale manufacturing: perspectives and measures. Int J Extreme Manuf 2(3):030201

4. Fang FZ, Xu FF (2018) Recent advances in micro/nano-cutting: effect of tool edge and material properties. Nanomanuf Metrol $1: 4-31$

5. Fang FZ, Wu H, Liu Y (2005) Modelling and experimental investigation on nanometric cutting of monocrystalline silicon. Int $\mathrm{J}$ Mach Tools Manuf 45(15):1681-1686

6. Wang JS, Zhang XD, Fang FZ et al (2018) A numerical study on the material removal and phase transformation in the nanometric cutting of silicon. Appl Surf Sci 455:608-615

7. Xie WK, Fang FZ (2019) Cutting-based single atomic layer removal mechanism of monocrystalline copper: atomic sizing effect. Nanomanuf Metrol 2:241-252

8. Xie WK, Fang FZ (2020) Effect of tool edge radius on material removal mechanism in atomic and close-to-atomic scale cutting. Appl Surf Sci 504:144451
9. Xie WK, Fang FZ (2020) On the mechanism of dislocationdominated chip formation in cutting-based single atomic layer removal of monocrystalline copper. Int J Adv Manuf Technol 108:1587-1599

10. Xu NS, Tzeng Y, Latham RV (1993) Similarities in the "cold" electron emission characteristics of diamond coated molybdenum electrodes and polished bulk graphite surfaces. J Phys D Appl Phys 26:1776-1780

11. Thakre P (2012) Effect of surface roughness and radiation on graphite nozzle erosion in solid rocket motors. J Propul Power 28(2):448-451

12. Park JY, Jeong MH, Kim D et al (2013) Effects of the surface roughness of a graphite substrate on the interlayer surface roughness of deposited SiC layer. J Korean Ceram Soc 50(2):122-126

13. Burton T, Back T, Fairchild S et al (2017) Influence of surface roughness on secondary electron emission from graphite. J Vac Sci Technol A Vac Surf Films 35:041404

14. Fu H (2006) Research on graphite cutting mechanism. Guangdong University of Technology, Guangzhou

15. Xu PJ (2014) The mechanism of material removal in cutting graphite. South China University of Technology, Guangzhou

16. Wang MH, Yin WD, Wang B et al (2016) Influence of cutting parameters on cutting force of isotropy pyrolytic graphite. Mach Des Manuf 8:121-127

17. Wang MH, Wang W, Huang ST et al (2012) Study on the mechanism of diamond wear in precision cutting of isotropic pyrolytic graphite. Key Eng Mater 499:168-172

18. Lei X, Wang L, Shen B et al (2013) Effect of boron-doped diamond interlayer on cutting performance of diamond coated micro drills for graphite machining. Materials 6:3128-3138

19. Hashimoto M, Kanda K, Tsubokawa T (2018) Reduction of diamond-coated cutting tool wear during graphite cutting. Precis Eng $51: 186-189$

20. Wang JS, Zhang XD, Fang FZ et al (2020) Study on nano-cutting of brittle material by molecular dynamics using dynamic modeling. Comput Mater Sci 183:109851

21. Wang JS, Fang FZ (2021) Nanometric cutting mechanism of silicon carbide. CIRP Ann Manuf Technol 70(1):29-32

22. Plimpton S (1995) Fast parallel algorithms for short-range molecular dynamics. J Comput Phys 117(1):1-19

23. Stuart SJ, Tutein AB, Harrison JA (2000) A reactive potential for hydrocarbons with intermolecular interactions. J Chem Phys 112(14):6472-6486

24. Li R, Liu B, Gao F (2019) Molecular dynamics study of the material property changes induced by accumulated point defects in graphite. Nuclear Instrum Methods Phys Res B Beam Interact Mater Atoms 455:52-56

25. Erhart P, Albe K (2005) Analytical potential for atomistic simulations of silicon, carbon, and silicon carbide. Phys Rev B Condens Matter Mater Phys 71:035211

26. Toxvaerd S, Dyre JC (2011) Communication: Shifted forces in molecular dynamics. J Chem Phys 134:081102

27. Yan Y, Lei Y, Liu S (2018) Tensile responses of carbon nanotubesreinforced copper nanocomposites: Molecular dynamics simulation. Comput Mater Sci 151:273-277

28. Wang B, Liu D, Wang M et al (2015) Wear mechanism of single crystal diamond tool during cutting of isotropic pyrolytic graphite. J Synth Cryst 44(10):2862-2868

29. Pei Q, Zhang Y, Shenoy V (2010) A molecular dynamics study of the mechanical properties of hydrogen functionalized graphene. Carbon 48(3):898-904 


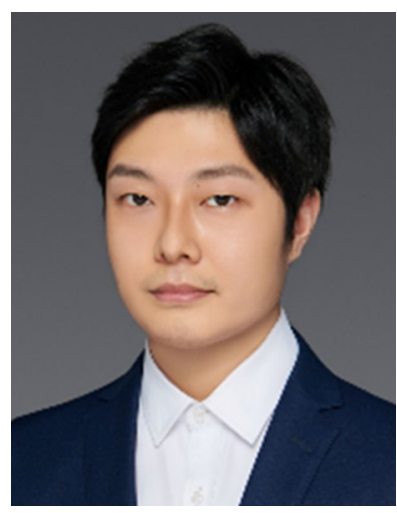

Jinshi Wang is a post-doctoral researcher working at the Laboratory of Micro/Nano Manufacturing Technology (MNMT), Tianjin University. His research focuses on nanometric machining and atomic and close-toatomic scale manufacturing (ACSM).

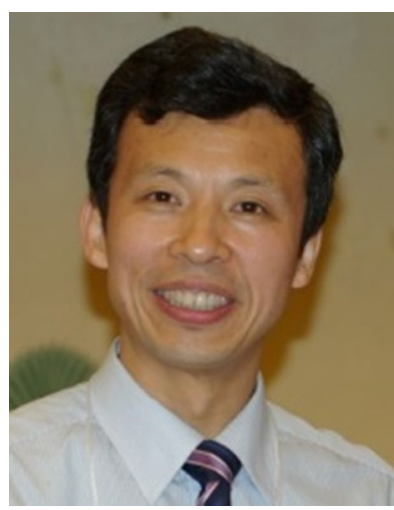

Fengzhou Fang is a joint professor working at Tianjin University and University College Dublin. His research focuses on both fundamental studies and application development in the areas of micro/nano machining, optical freeform design and manufacturing, ultra-precision machining and measurement, and ACSM benefiting a variety of industries in medical devices, bio-implants, optics and molds sectors.

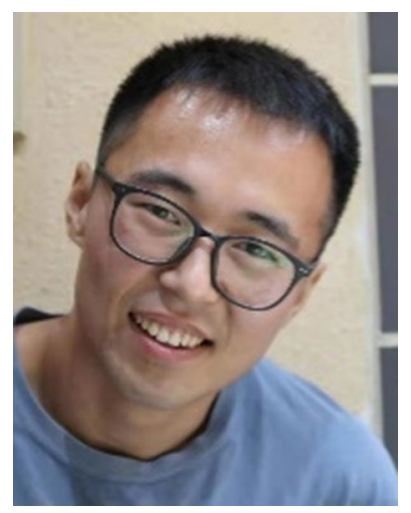

Luan Li completed his Bachelor degree in measuring and controlling technologies and instruments from Tianjin University. His research interests include mathematical modeling, software engineering and digital twin. 